International Journal of Modern Physics: Conference Series

(C) World Scientific Publishing Company

\title{
NEW MODEL FOR NUCLEON GENERALIZED PARTON DISTRIBUTIONS
}

\author{
ANATOLY V. RADYUSHKIN* \\ Physics Department, Old Dominion University, Norfolk, VA 23529, USA \\ and \\ Thomas Jefferson National Accelerator Facility, Newport News, VA 23606, USA \\ radyush@jlab.org
}

\begin{abstract}
We describe a new type of models for nucleon generalized parton distributions (GPDs) $H$ and $E$. They are heavily based on the fact nucleon GPDs require to use two forms of double distribution (DD) representations. The outcome of the new treatment is that the usual "DD+D-term" construction should be amended by an extra term, $\xi E_{+}^{1}(x, \xi)$ which has the DD structure $\alpha / \beta e(\beta, \alpha)$, with $e(\beta, \alpha)$ being the DD that generates GPD $E(x, \xi)$. We found that this function, unlike the $D$-term, has support in the whole $-1 \leq x \leq 1$ region. Furthermore, it does not vanish at the border points $|x|=\xi$.
\end{abstract}

PACS numbers:11.10.-z,12.38.-t,13.60.Fz

\section{Introduction}

Building theoretical models for Generalized Parton Distributions (GPDs) [1, 2, 3, $4]$ is an inherent part of their studies. These models should satisfy several nontrivial requirements that follow from most general principles of quantum field theory. In this context, one could mention polynomiality ${ }^{5}$, positivity ${ }^{6,7,8}$, hermiticity $^{1}$, time reversal invariance ${ }^{5}$, etc. In particular, the polynomiality property states that $x^{n}$ moment of a GPD $H(x, \xi ; t)$ must be a polynomial in $\xi$ (of order not higher than $n+1)$. An efficient way to impose the polynomiality constraint is to incorporate Double Distributions (DDs) $F(\beta, \alpha ; t)[1,3,9,8]$ as the first step of constructing GPDs. (Another way to satisfy the polynomiality condition is to use "dual parameterization" $[10,11,12,13,14])$.

Within the DD approach, the problem of constructing a model for a GPD thus converts into a problem of building a model for the relevant DD. Modeling of double distributions $F(\beta, \alpha ; t)$ is based on their physical interpretation: DDs behave like usual parton distribution functions (PDFs) with respect to the first variable $\beta$, as a meson distribution amplitude (DA) with respect to the second variable $\alpha$, and as a form factor with respect to the invariant momentum transfer $t$. Since the $t$-dependence is not crucial for the polynomiality property, it makes sense to

*Also at Bogoliubov Laboratory of Theoretical Physics, JINR, 141980 Dubna, Russian Federation 
concentrate first on the $\beta, \alpha$ dependence of DDs and consider for simplicity the formal $t=0$ limit.

In the $t=0$ limit, the factorized DD ansatz (FDDA) $[8,15]$ proposes to build a model $\mathrm{DD} F(\beta, \alpha)$ as a product of the usual parton density $f(\beta)$ and a profile function $h(\beta, \alpha)$ that has an $\alpha$-shape of a meson DA. However, the simplest $F(\beta, \alpha) \rightarrow H(x, \xi)$ transition formula ${ }^{16}$, derived from the analysis of scalar composite operators in scalar field theories, does not produce the highest, $(n+1)^{\text {st }}$ power of the skewness parameter $\xi$ that is required for vector operators. It was noted $^{17}$, that for pion GPDs one should use a parametrization involving two DDs, with the second $\mathrm{DD} G(\beta, \alpha)$ capable of generating the required $\xi^{n+1}$ power. It was also argued ${ }^{17}$ that the second DD $G(\beta, \alpha)$ may be reduced to a function $D(\alpha)$ of just one variable, the $D$-term, that is solely responsible for the $\xi^{n+1}$ contribution, and thus one arrives at "DD plus D" decomposition.

Since the pion is described by just one GPD $H(x, \xi ; t)$, the use of two DDs, $F(\beta, \alpha)$ and $G(\beta, \alpha)$ looks redundant. Indeed, it was observed ${ }^{18}$ that GPD $H$ is determined by $\partial F(\beta, \alpha) / \partial \beta+\partial G(\beta, \alpha) / \partial \alpha$ combination, so that one can reshuffle terms between $F$ and $G$ changing them by a "gauge transformation" 18 which renders above combination of DDs unchanged. The $D$ term is given by the $\beta$-integral of $G(\beta, \alpha)$, and is not changed by gauge transformations. Choosing $G(\beta, \alpha)=$ $\delta(\beta) D(\alpha)$ corresponds to "Polyakov-Weiss" gauge, in which non-D-term part is given by just one function $F_{D}(\beta, \alpha)$. Another choice, "one-DD" gauge corresponds to $F_{O}(\beta, \alpha)=\beta \mathcal{F}(\beta, \alpha), G_{O}(\beta, \alpha)=\alpha \mathcal{F}(\beta, \alpha)$, where $\mathcal{F}(\beta, \alpha)$ is the DD of one-DD gauge representation for GPD $H$. The one-DD gauge naturally appears for matrix elements of twist- 2 vector operators ${ }^{19}$, in which the Lorentz index $\mu$ of the bilocal vector operator $\mathcal{O}_{\mu}(0, z)$ is symmetrized with indices $\mu_{i}$ of the derivatives $D_{\mu_{i}}$ in the corresponding local twist-2 operators $O_{\mu \mu_{1} \ldots \mu_{i} \ldots \mu_{n}}$.

Applying FDDA to a one-DD representation is complicated by the fact that one should now reconstruct GPD from $f(\beta) / \beta$. The extra $1 / \beta$ factor combined with the Regge $\beta^{-a}$ singularity of the parton density $f(\beta)$ results in a non-integrable singularity for $\beta=0$. A pion GPD model based on one-DD gauge was built in our paper [20], (see also [21]). There we have separated DD $\mathcal{F}(\beta, \alpha)$ in the "plus" part $[f(\beta, \alpha)]_{+}$that gives zero after integration over $\beta$, and the $D$-term part $\delta(\beta) D(\alpha) / \alpha$. For DDs singular in small- $\beta$ region, such a separation serves also as a renormalization prescription substituting a formally divergent integral over $\beta$ by the "observable" $D$-term. Given the ambiguity of DDs involved, one should choose the gauge in which the FDDA is applied. Originally, FDDA in case of pion was used for the "DD+D" decomposition, or in Polyakov-Weiss gauge.

For the nucleon, a straightforward parametrization involves 3 double distributions ${ }^{17,22,23}$, one of which is redundant (there are only two GPDs, $H(x, \xi ; t)$ and $E(x, \xi ; t))$, i.e., as in pion case, one can perform gauge transformations that do not change the total sum. Again, imposing symmetrization of indices involved in the definition of local twist-2 operators, one obtains a natural parametrization in terms of just two DDs $\mathcal{A}$ and $\mathcal{B}$ [24]. They correspond to GPDs $A$ and $B$ [24] that 
do not coincide with standard GPDs $H$ and $E$.

The analog of "DD+D" construction for the nucleon has the structure $H_{D D}+D$ for GPD $H$ and $E_{D D}-D$ for GPD $E$. In fact, the combination that has the simplest DD representation is $A=H+E$, whereas $B=-E$ has a DD representation identical in structure to one-DD representation of the pion case ${ }^{24,25}$. Using FDDA, one would reconstruct $A$ from the forward function $f(x)+e(x)$ (which, roughly speaking, is equivalent to taking $H+E=H_{D D}+E_{D D}$ ), while $B$ is reconstructed from $-e(x) / x$, which requires special treatment of the $x=0$ singularity. The result for $E$ may be written as $E=E_{D D}+\xi E_{+}^{1}-D[25]$, where $E_{+}^{1}(x, \xi)$ is an extra term specific to the one-DD parametrization. Unlike the $D$-term, it does not vanish at the border point $x=\xi$. As a result, $H=H_{D D}-\xi E_{+}^{1}+D$, i.e. GPD $H$ also acquires an extra term affecting its value at border point $x=\xi$.

In the present paper, we describe recent application ${ }^{25}$ of the technique of Ref. [20] (see also [21]) for building models of nucleon GPDs $H(x, \xi)$ and $E(x, \xi)$. In Sect. 2, we give a short review of the basic facts about DDs, GPDs and $D$-term, using a toy model with scalar quarks, that allows to illustrate essential features of GPD theory avoiding complications related to spin. In Sect. 3, we describe the theory of pion GPD $H(x, \xi)$, presenting the results of Ref. [20] in a form suitable for generalization onto the nucleon case. In Sect. 4, we recall the basic ideas of the factorized DD Ansatz of Refs. [8, 15]. In Sect. 5, we use the formalism described in previous sections for building DD models for nucleon GPDs $H(x, \xi)$ and $E(x, \xi)$.

\section{Basics of theory for DDs and GPDs}

\subsection{Matrix elements and DDs}

Parton distributions provide a convenient way to parametrize matrix elements of local operators that accumulate information about hadronic structure. Various types of distributions differ by the nature of the matrix elements involved. In particular, to define GPDs, one starts with non-forward matrix elements $\langle P+r / 2|\ldots| P-r / 2\rangle$, with $P$ being the average of the initial and final hadron momenta, and $r$ being their difference. In scalar case (which illustrates many essential features without irrelevant complications) we have

$$
\begin{aligned}
\left\langle P+r / 2\left|\psi(0)\left\{\stackrel{\leftrightarrow}{\partial}_{\mu_{1}} \ldots \stackrel{\leftrightarrow}{\partial}_{\mu_{n}}\right\} \psi(0)\right| P-r / 2\right\rangle= & \sum_{l=0}^{n-1} A_{n l}\left\{P_{\mu_{1}} \ldots P_{\mu_{n-l}} r_{\mu_{n-l+1}} \ldots r_{\mu_{n}}\right\} \\
& +A_{n n}\left\{r_{\mu_{1}} \ldots r_{\mu_{n}}\right\}
\end{aligned}
$$

The notation $\{\ldots\}$ indicates the symmetric-traceless part of the enclosed tensor. Since two vectors are involved, we have $n+1$ distinct tensor structures differing in the number $l$ of $r$ factors involved. In the forward $r=0$ limit, only the $A_{n_{0}}$ coefficients are visible. Another extreme case is $l=n$, corresponding to the tensor $\left\{r_{\mu_{1}} \ldots r_{\mu_{n}}\right\}$ built solely from the $r$ momentum.

The forward $r=0$ limit corresponds to matrix elements defining usual parton 
distribution $f(x)$ as a function whose moments produce $A_{n 0}$ :

$$
\int_{-1}^{1} f(x) x^{n} d x=A_{n_{0}} .
$$

The parton interpretation is that $f(x)$ it describes a parton with momentum $x P$. In terms of matrix elements of operators on the light cone one has

$$
\langle P|\psi(-z / 2) \psi(z / 2)| P\rangle=\int_{-1}^{1} f(x) e^{-i x(P z)} d x+\mathcal{O}\left(z^{2}\right) .
$$

In non-forward case, the parton carries fractions of both $P$ and $r$ momenta. Treating the average $\bar{k}_{\mu}=\left(k_{\mu}+k_{\mu}^{\prime}\right) / 2$ momentum of the initial and final quark as $\beta P+\alpha r / 2$ one can define the double distribution (DD) $[1,3,9,8]$ as a function whose $\beta^{n-l} \alpha^{l}$ moments are proportional to the coefficients $A_{n l}$. This definition results in the "DD parameterization"

$$
\left\langle P-\frac{r}{2}\left|\psi\left(-\frac{z}{2}\right) \psi\left(\frac{z}{2}\right)\right| P+\frac{r}{2}\right\rangle=\int_{\Omega} F(\beta, \alpha) e^{-i \beta(P z)-i \alpha(r z) / 2} d \beta d \alpha+\mathcal{O}\left(z^{2}\right) .
$$

The support region $\Omega$ is given by the rhombus $|\alpha|+|\beta| \leq 1[1,3,15]$.

\subsection{Introducing GPDs and D-term}

Another parametrization of the non-forward matrix element is in terms of generalized parton distributions. In scalar case GPDs are defined by

$$
\langle P-r / 2|\psi(-z / 2) \psi(z / 2)| P+r / 2\rangle \int_{-1}^{1} e^{-i x(P z)} H(x, \xi) d x+\mathcal{O}\left(z^{2}\right),
$$

and relation between GPD and DD functions is given by

$$
H(x, \xi)=\int_{\Omega} F(\beta, \alpha) \delta(x-\beta-\xi \alpha) d \beta d \alpha .
$$

In the forward limit $\xi=0$, GPD $H(x, \xi)$ converts into the usual parton distribution $f(x)$. Using DDs, we may write

$$
f(x)=\int_{-1+|x|}^{1-|x|} F(x, \alpha) d \alpha .
$$

Thus, the forward distributions $f(x)$ are obtained by integrating DDs over vertical lines $\beta=x$ in the $(\beta, \alpha)$ plane. As discussed above, $f(x)$ is defined through the coefficients $A_{n 0}$ corresponding to tensors without $r$ factors. Similarly, one can treat the $A_{n n}$ coefficients, corresponding to tensors without $P$ factors, as the moments of another function $D(\alpha)$

$$
\int_{-1}^{1} D(\alpha)(\alpha / 2)^{n} d \alpha=A_{n n}
$$


the $D$-term ${ }^{17}$. From the definition of DD (4), it follows that

$$
D(\alpha)=\int_{-1+|\alpha|}^{1-|\alpha|} F(\beta, \alpha) d \beta,
$$

i.e., $D$-term $D(\alpha)$ is obtained from $\mathrm{DD} F(\beta, \alpha)$ by integration over horizontal lines in the $\{\beta, \alpha\}$ plane.

\section{Pion DDs and GPDs}

\subsection{Two-DD representation}

$D$-term was introduced first ${ }^{17}$ for pion GPDs related to vector bilocal operator

$$
\begin{aligned}
& \left.\left\langle P-r / 2\left|\bar{\psi}(-z / 2) \gamma_{\mu} \psi(z / 2)\right| P+r / 2\right\rangle\right|_{\text {twist }-2} \\
& =2 P_{\mu} f\left((P z),(r z), z^{2}\right)+r_{\mu} g\left((P z),(r z), z^{2}\right) .
\end{aligned}
$$

This suggests a parametrization with two DDs corresponding to $f$ and $g$ functions ${ }^{17}$,

$$
\begin{aligned}
z^{\mu}\left\langle P-r / 2\left|\bar{\psi}(-z / 2) \gamma_{\mu} \psi(z / 2)\right| P+r / 2\right\rangle= & \int_{\Omega} e^{-i \beta(P z)-i \alpha(r z) / 2}[2(P z) F(\beta, \alpha) \\
& +(r z) G(\beta, \alpha)] d \beta d \alpha+\mathcal{O}\left(z^{2}\right)
\end{aligned}
$$

Then GPDs are given by a DD representation

$$
H(x, \xi)=\int_{\Omega}[F(\beta, \alpha)+\xi G(\beta, \alpha)] \delta(x-\beta-\xi \alpha) d \beta d \alpha,
$$

that involves two DDs: $F(\beta, \alpha)$ and $G(\beta, \alpha)$. The highest power $\xi^{n+1}$ for the $x^{n}$ moment of $H(x, \xi)$ is given now by the $G$ term, which one can separate $G(\beta, \alpha)=$ $[G(\beta, \alpha)]_{+}+\delta(\beta) D(\alpha)$ into a "plus" part

$$
[G(\beta, \alpha)]_{+}=G(\beta, \alpha)-\delta(\beta) \int_{-1+|\alpha|}^{1-|\alpha|} G(\gamma, \alpha) d \gamma
$$

and $D$-term

$$
D(\alpha)=\int_{-1+|\alpha|}^{1-|\alpha|} G(\beta, \alpha) d \beta .
$$

As a result, $H(x, \xi)=F(x, \xi)+\xi G_{+}(x, \xi)+\operatorname{sgn}(\xi) D(x / \xi)$, where

$$
F(x, \xi)=\int_{\Omega} F(\beta, \alpha) \delta(x-\beta-\xi \alpha) d \beta d \alpha
$$

and

$$
G_{+}(x, \xi)=\int_{\Omega} G(\beta, \alpha)[\delta(x-\beta-\xi \alpha)-\delta(x-\xi \alpha)] d \beta d \alpha .
$$

The forward distribution $f(x)$ in two-DD formulation is obtained from the DD $F$, as in (25). Thus, $D$-term $D(\alpha)$ and PDF $f(x)$ are obtained from different functions. 


\subsection{One-DD representation}

Note that the Dirac index $\mu$ is symmetrized in the local twist-two operators $\bar{\psi}\left\{\gamma_{\mu} \stackrel{\leftrightarrow}{\partial}_{\mu_{1}} \ldots \stackrel{\leftrightarrow}{\partial}_{\mu_{n}}\right\} \psi$ with $\mu_{i}$ indices of the derivatives. In Ref. [19], it was shown that it also produces the factor $\beta P_{\mu}+\alpha r_{\mu} / 2$. The result is a representation

$$
2(P z) F(\beta, \alpha)+(r z) G(\beta, \alpha)=[2 \beta(P z)+\alpha(r z)] f(\beta, \alpha) .
$$

It corresponds to $F(\beta, \alpha)=\beta f(\beta, \alpha)$ and $G(\beta, \alpha)=\alpha f(\beta, \alpha)$. Thus, one deals formally with just one $\mathrm{DD} f(\beta, \alpha)$. The two-DD representation (12) converts into

$$
\begin{aligned}
H(x, \xi) & =\int_{\Omega}(\beta+\xi \alpha) f(\beta, \alpha) \delta(x-\beta-\xi \alpha) d \beta d \alpha \\
& =x \int_{\Omega} f(\beta, \alpha) \delta(x-\beta-\xi \alpha) d \beta d \alpha
\end{aligned}
$$

in the "one-DD" formulation. The $D$-term in the one- $\mathrm{DD}$ case is given by

$$
D(\alpha)=\alpha \int_{-1+|\alpha|}^{1-|\alpha|} f(\beta, \alpha) d \beta
$$

and one may write $f(\beta, \alpha)$ as a sum

$$
f(\beta, \alpha)=[f(\beta, \alpha)]_{+}+\delta(\beta) \frac{D(\alpha)}{\alpha}
$$

of its "plus" part and $D$-term part $\delta(\beta) D(\alpha) / \alpha$. For the GPD $H(x, \xi)$, the "DD + D" separation corresponds to the representation

$$
H(x, \xi) \equiv H_{+}(x, \xi)+\operatorname{sgn}(\xi) D(x / \xi),
$$

where

$$
\frac{H_{+}(x, \xi)}{x} \equiv \int_{\Omega} f(\beta, \alpha)[\delta(x-\beta-\xi \alpha)-\delta(x-\xi \alpha)] d \beta d \alpha
$$

Using $f(\beta, \alpha)=F(\beta, \alpha) / \beta$ we may rewrite

$$
\begin{aligned}
& H(x, \xi)=\int_{\Omega} F(\beta, \alpha) \delta(x-\beta-\xi \alpha) d \beta d \alpha \\
& +\xi \int_{\Omega} \frac{\alpha F(\beta, \alpha)}{\beta}[\delta(x-\beta-\xi \alpha)-\delta(x-\xi \alpha)] d \beta d \alpha+\operatorname{sgn}(\xi) D(x / \xi) \\
& \equiv F_{D D}(x, \xi)+\xi F_{+}^{1}(x, \xi)+\operatorname{sgn}(\xi) D(x / \xi),
\end{aligned}
$$

where $F_{D D}(x, \xi)$ is GPD constructed from $\mathrm{DD} F(\beta, \alpha)$ by the same formula as in scalar case. Another term

$$
F_{+}^{1}(x, \xi) \equiv \int_{\Omega}\left(\frac{\alpha}{\beta} F(\beta, \alpha)\right)_{+} \delta(x-\beta-\xi \alpha) d \beta d \alpha
$$

is a GPD built from the "plus" part of the $\mathrm{DD} \alpha F(\beta, \alpha) / \beta$. The latter, of course, may be written as $G(\beta, \alpha)$, but in the spirit of the one-DD formulation, one may wish to express the results in terms of just one function $F(\beta, \alpha)$. 


\section{Factorized DD Ansatz}

In the forward limit $\xi=0$, GPD $H(x, \xi)$ converts into the usual parton distribution $f(x)$. In the one-DD formulation, we may write

$$
f(x)=x \int_{-1+|x|}^{1-|x|} f(x, \alpha) d \alpha .
$$

This reduction formula (25) suggests the factorized DD Ansatz

$$
f(\beta, \alpha)=h(\beta, \alpha) f(\beta) / \beta,
$$

where $f(\beta)$ is the forward distribution, while $h(\beta, \alpha)$ is a normalized profile in the $\alpha$ direction. The profile function should be symmetric with respect to $\alpha \rightarrow-\alpha$ because DDs $f(\beta, \alpha)$ are even in $\alpha[32,15]$. For a fixed $\beta$, the function $h(\beta, \alpha)$ describes how the longitudinal momentum transfer $r^{+}$is shared between the two partons. Hence, it is natural to expect that the shape of $h(\beta, \alpha)$ should look like a symmetric meson distribution amplitude (DA) $\varphi(\alpha)$. As a model profile function one can use

$$
h^{(N)}(\beta, \alpha)=\frac{\Gamma(2 N+2)}{2^{2 N+1} \Gamma^{2}(N+1)} \frac{\left[(1-|\beta|)^{2}-\alpha^{2}\right]^{N}}{(1-|\beta|)^{2 N+1}},
$$

where the width of $\alpha$-shape is governed by the parameter $N$.

In Fig. 1 we show the simplest GPD $F_{D D}(x, \xi)$ built from the model $F(\beta, \alpha)=f(\beta) h^{(1)}(\beta, \alpha)$, with the forward distribution $f^{\bmod }(x)=(1-x)^{3} / \sqrt{x}$ and $N=1$ profile function The model forward function was chosen in the form reproducing the $x \rightarrow 1$ behavior of the nucleon parton distributions and the $\sim x^{-0.5}$ Regge behavior of valence part of quark distributions for small $x$, which was taken for simplicity, though the GPD shown corresponds to the $C$-even component (the full function is antisymmetric in $x$, and only the $x \geq 0$ part is shown).

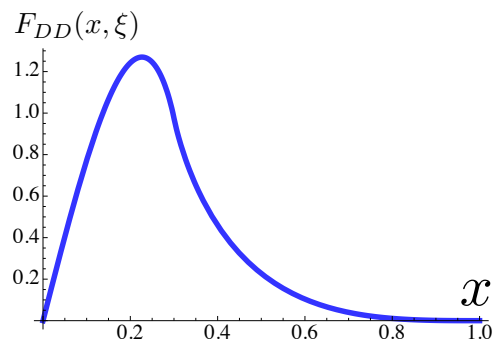

Fig. 1. $\operatorname{GPD} F_{D D}(x, \xi)$ for $\xi=0.3$.

\section{Nucleon GPDs}

\subsection{Definitions of DDs and GPDs}

In the nucleon case, for unpolarized target, one can parametrize

$$
\begin{aligned}
\left\langle p^{\prime}|\bar{\psi}(-z / 2) \not \psi(z / 2)| p\right\rangle & \left.\right|_{\text {twist }-2}=\int_{\Omega} e^{-i \beta(P z)-i \alpha(r z) / 2}\left[\bar{u}\left(p^{\prime}\right) \not u(p) a(\beta, \alpha)\right. \\
+ & \left.\frac{\bar{u}\left(p^{\prime}\right) u(p)}{2 M_{N}}[2 \beta(P z)+\alpha(r z)] b(\beta, \alpha)\right] d \beta d \alpha+\mathcal{O}\left(z^{2}\right) .
\end{aligned}
$$

Here, the functions $a, b$ are DDs corresponding to the combinations $A=H+E$ and $B=-E$ of usual GPDs $H$ and $E$ (see Ref. [24]). These GPDs may be expressed in 
terms of relevant DDs as

$$
A(x, \xi)=\int_{\Omega} a(\beta, \alpha) \delta(x-\beta-\xi \alpha) d \beta d \alpha
$$

and

$$
B(x, \xi)=x \int_{\Omega} b(\beta, \alpha) \delta(x-\beta-\xi \alpha) d \beta d \alpha .
$$

We have two different types of relations between GPDs and DDs: $A(x, \xi)$ is obtained from its $\mathrm{DD} a(\beta, \alpha)$ just like in the simplest scalar case, while $B(x, \xi)$ is calculated from $b(\beta, \alpha)$ using the formula with the one-DD representation structure. In the forward limit, we have $A(x, 0)=H(x, 0)+E(x, 0)=f(x)+e(x)$ and $B(x, 0)=$ $-E(x, 0)=-e(x)$. These reduction formulas suggest the model representation $a(\beta, \alpha)=f(\beta, \alpha)+e(\beta, \alpha)$ and $b(\beta, \alpha)=-e(\beta, \alpha) / \beta$. Because of possible singularity of $e(\beta, \alpha) / \beta$ at $\beta=0$, we write it in the " $\mathrm{DD}_{+}+D$ " representation:

$$
b(\beta, \alpha)=-\left(\frac{e(\beta, \alpha)}{\beta}\right)_{+}+\delta(\beta) \frac{D(\alpha)}{\alpha},
$$

where $D(\alpha)$ is the $D$-term.

\subsection{General results for GPDs}

As a result, we have

$$
\begin{aligned}
H(x, \xi)= & A(x, \xi)+B(x, \xi)=\int_{\Omega}[f(\beta, \alpha)+e(\beta, \alpha)] \delta(x-\beta-\xi \alpha) d \beta d \alpha \\
& -x \int_{\Omega}\left[\left(\frac{e(\beta, \alpha)}{\beta}\right)_{+}-\delta(\beta) \frac{D(\alpha)}{\alpha}\right] \delta(x-\beta-\xi \alpha) d \beta d \alpha \\
& =F_{D D}(x, \xi)+E_{D D}(x, \xi)-E_{+}(x, \xi)+\operatorname{sgn}(\xi) D(x / \xi),
\end{aligned}
$$

where $F_{D D}(x, \xi)$ and $E_{D D}(x, \xi)$ are build from DDs by simplest formulas not involving a division by $\beta$ factors, while

$$
\frac{E_{+}(x, \xi)}{x}=\int_{\Omega}\left(\frac{e(\beta, \alpha)}{\beta}\right)_{+} \delta(x-\beta-\xi \alpha) d \beta d \alpha
$$

has the structure of a one-DD representation. Since $E_{+}(x, \xi) / x$ is built from the "plus" part of a DD it should satisfy

$$
\int_{-1}^{1} E_{+}(x, \xi) d x=\int_{\Omega}\left[\frac{e(\beta, \alpha)}{\beta}\right]_{+} d \beta d \alpha=0 .
$$

Being (for $C$-even combination) an even function of $x$, the function $E_{+}(x, \xi) / x$ also gives zero after integration over the $(0,1)$ interval.

\subsection{Modeling GPDs}

To illustrate the structure of $E_{+}(x, \xi)$, we show it in Fig. 2 using the model based 
on $e(\beta, \alpha)=e(\beta) h^{(1)}(\beta, \alpha)$ with $N=1$ profile function and the same forward distribution $e(x)=(1-x)^{3} / \sqrt{x}$ that was used to model $F_{D D}$ above. Again, we have in mind the $C$-even, quark+antiquark part of the distribution, and valence-type functional form is used to simplify the illustration. One can see that $E_{+}(x, \xi)$ is a regular function, and vanishing of $E_{+}(x, \xi) / x$ integral is due to compensation over positive and negative parts rather than because of subtraction of a $\delta(x)$ term.

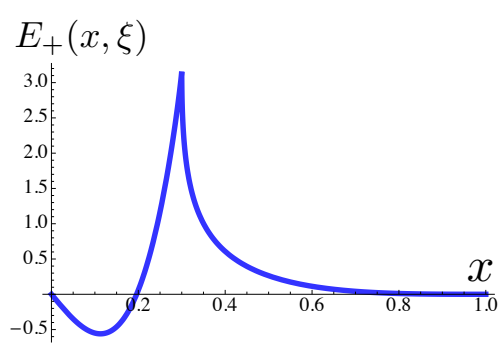

Fig. 2. $\operatorname{GPD} E_{+}(x, \xi)$ for $\xi=0.3$.

In a more realistic modeling, one should adjust normalization of $e(x)$ to reflect its relation to the anomalous magnetic moment. Also, the fits of the nucleon elastic form factors ${ }^{35}$ suggest for $e(x)$ a higher power of $(1-x)$. However, our aim while showing the curves in the present paper is just to illustrate the qualitative features of various GPD models, so we will stick to the same generic forward function both for $f(x)$ and $e(x)$. The function $E_{+}(x, \xi)$ may be represented as

$$
\begin{gathered}
E_{+}(x, \xi)=x \int_{\Omega} \frac{e(\beta, \alpha)}{\beta}[\delta(x-\beta-\xi \alpha)-\delta(x-\xi \alpha)] d \beta d \alpha \\
\equiv=E_{D D}(x, \xi)+\xi E_{+}^{1}(x, \xi),
\end{gathered}
$$

where

$$
E_{+}^{1}(x, \xi) \equiv \int_{\Omega}\left(\frac{\alpha}{\beta} e(\beta, \alpha)\right)_{+} \delta(x-\beta-\xi \alpha) d \beta d \alpha .
$$

Summarizing, GPD $E_{+}$is obtained from the naive $E_{D D}$ function by adding to it the $\xi E_{+}^{1}(x, \xi)$ term, which results in a rather nontrivial non-monotonic behavior of the $E_{+}$function. To get the full GPD $E$, one should subtract also the $D$-term contribution:

$$
E(x, \xi)=E_{+}(x, \xi)-\operatorname{sgn}(\xi) D(x / \xi)=E_{D D}(x, \xi)+\xi E_{+}^{1}(x, \xi)-\operatorname{sgn}(\xi) D(x / \xi) .
$$
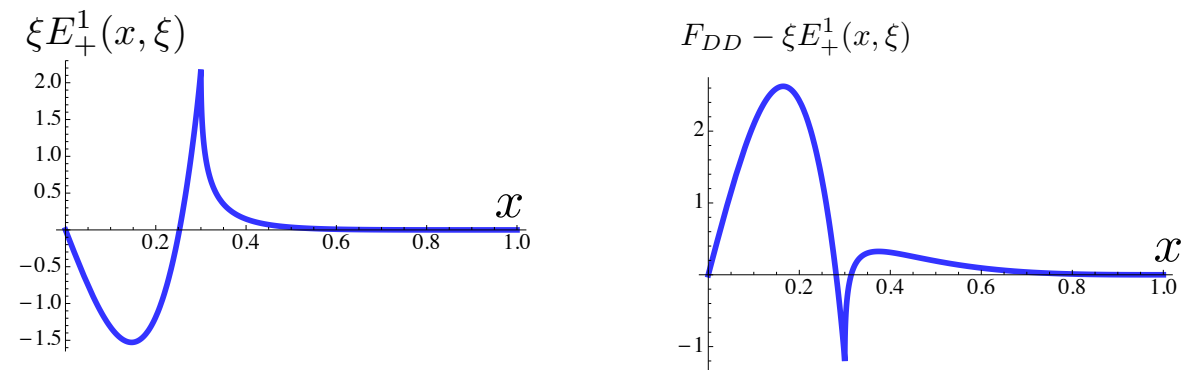

Fig. 3. Left: Function $\xi E_{+}^{1}(x, \xi)$; Right: Model GPD $H(x, \xi)$ (without $D$-term); both for $\xi=0.3$. 
For GPD $H$, we then have

$$
H(x, \xi)=F_{D D}(x, \xi)-\xi E_{+}^{1}(x, \xi)+\operatorname{sgn}(\xi) D(x / \xi) .
$$

Thus, one should subtract $\xi E_{+}^{1}(x, \xi)$ from the naive $F_{D D}$ function and then add the $D$-term contribution.

\section{Summary}

Summarizing, in this paper we described the model for nucleon GPDs $H$ and $E$ developed in Ref. [25]. The result for GPD $H$ obtained within this model differs from the "old-fashioned" DD $+\mathrm{D}$ model by an extra $-\xi E_{+}^{1}(x, \xi)$ term constructed from the $\mathrm{DD} e(\beta, \alpha)$ corresponding to the GPD $E(x, \xi)$. The inclusion of such a term modifies the original DD-based term $F_{D D}(x, \xi)$ at the border points $|x|=\xi$ and outside the central $|x / \xi| \leq 1$ region, which may have strong phenomenological consequences.

\section{Acknowledgements}

I thank H. Moutarde and A. Tandogan for discussions, and C. Mezrag for correspondence.

Authored by Jefferson Science Associates, LLC under U.S. DOE Contract No. DE-AC05-06OR23177. The U.S. Government retains a non-exclusive, paid-up, irrevocable, world-wide license to publish or reproduce this manuscript for U.S. Government purposes.

\section{References}

1. D. Mueller, D. Robaschik, B. Geyer, F. M. Dittes and J. Horejsi, Fortschr. Phys. 42, p. 101 (1994).

2. X.-D. Ji, Phys. Rev. Lett. 78, 610 (1997).

3. A. V. Radyushkin, Phys. Lett. B380, 417 (1996).

4. J. C. Collins, L. Frankfurt and M. Strikman, Phys. Rev. D56, 2982 (1997).

5. X.-D. Ji, J. Phys. G24, 1181 (1998).

6. A. D. Martin and M. G. Ryskin, Phys. Rev. D57, 6692 (1998).

7. B. Pire, J. Soffer and O. Teryaev, Eur. Phys. J. C8, 103 (1999).

8. A. V. Radyushkin, Phys. Rev. D59, p. 014030 (1999).

9. A. V. Radyushkin, Phys. Lett. B385, 333 (1996).

10. M. V. Polyakov and A. G. Shuvaev, hep-ph/0207153 (2002).

11. M. V. Polyakov, arXiv:0711.1820 [hep-ph] (2007).

12. M. V. Polyakov, Phys. Lett. B659, 542 (2008).

13. K. M. Semenov-Tian-Shansky, Eur. Phys. J. A36, 303 (2008).

14. M. V. Polyakov and K. M. Semenov-Tian-Shansky, Eur. Phys. J. A40, 181 (2009).

15. A. V. Radyushkin, Phys. Lett. B449, 81 (1999).

16. A. V. Radyushkin, Phys. Rev. D56, 5524 (1997).

17. M. V. Polyakov and C. Weiss, Phys. Rev. D60, p. 114017 (1999).

18. O. V. Teryaev, Phys. Lett. B510, 125 (2001). 
19. A. V. Belitsky, D. Mueller, A. Kirchner and A. Schafer, Phys. Rev. D64, p. 116002 (2001).

20. A. Radyushkin, Phys.Rev. D83, p. 076006 (2011).

21. A. V. Radyushkin, Int.J.Mod.Phys.Conf.Ser. 20, 251 (2012).

22. B. Tiburzi, W. Detmold and G. Miller, Phys.Rev. D70, p. 093008 (2004).

23. C. Mezrag, H. Moutarde and F. Sabatié, Phys. Rev. D 88, 014001 (2013).

24. A. V. Belitsky and A. V. Radyushkin, Phys. Rept. 418, 1 (2005).

25. A. Radyushkin, Phys.Rev. D87, p. 096017 (2013).

26. X.-D. Ji, Phys. Rev. D55, 7114 (1997).

27. O. V. Teryaev, hep-ph/0510031 (2005).

28. I. V. Anikin and O. V. Teryaev, Phys. Rev. D76, p. 056007 (2007).

29. M. Diehl and D. Y. Ivanov, Eur. Phys. J. C52, 919 (2007).

30. A. P. Szczepaniak, J. T. Londergan and F. J. Llanes-Estrada, Acta Phys. Polon. B40, 2193 (2009).

31. I. I. Balitsky and V. M. Braun, Nucl. Phys. B311, 541 (1989).

32. L. Mankiewicz, G. Piller and T. Weigl, Eur.Phys.J. C5, 119 (1998).

33. I. V. Musatov and A. V. Radyushkin, Phys. Rev. D61, p. 074027 (2000).

34. A. V. Radyushkin, e-print [hep-ph/0101225] (2000).

35. M. Guidal, M. Polyakov, A. Radyushkin and M. Vanderhaeghen, Phys.Rev. D72, p. 054013 (2005). 